\title{
A Faster Magnetic Contour Matching Algorithm
}

\author{
Lihua Chen \\ Xi'an Research Institute of High-tech \\ Shaanxi, China \\ e-mail: clhhxl@163.com \\ *Research supported by The Industrial Research Plan of \\ Shaanxi province under grant \#2012GY2-34. \\ Yanli Yang \\ Xi'an Research Institute of High-tech \\ Shaanxi, China \\ e-mail:58580300@qq.com
}

\author{
Zhiguo Liu \\ Xi'an Research Institute of High-tech \\ Shaanxi, China \\ e-mail: lzgc@163.com
}

\begin{abstract}
A new magnetic contour matching (MAGCOM) algorithm is proposed, which reduces computation by searching the solutions in $\delta$-neighborhood of measurement. This algorithm can be used in geomagnetism aided navigation to improve real-time practicability even there are large errors. Effect of the algorithm is evaluated by simulation. The results show that this algorithm has advantage in time-cost, and doesn't lead to matching probability and position error worse observably.
\end{abstract}

Keywords- magnetic contour matching; $\delta$-neighborhood; geomagnetism aided navigation; real-time practicability; timecost

\section{INTRODUCTION}

Geomagnetism aided navigation (GAN) is the technique using variations in geomagnetic field to estimate the position, which has been a focus of navigation field in recent years ${ }^{[1-4]}$. Being similar to terrain-aided navigation (TAN), the existed GAN algorithms stem from TAN, which has two means: magnetic contour matching (MAGCOM) and geomagnetic filter. While researches show that matching has no accumulated errors, and can be used in chopping mode and may obtain higher precision than filter ${ }^{[4]}$.magnetic matching becomes the main trend gradually.

The essential of MAGCOM is registration between reference map and real-time sequence measurements of geomagnetic field intensity. The estimated path is the one in which reference intensity is the closest to real-time sequence. To find out the "closest one", we usually compute all possible data in the search area. Therefore, the existed geomagnetic matching method, MAGCOM, will be in the trouble of long time consuming in large search area. A searching strategy which can reduce scope of search according to the real-time measured data at all times during the search course of possible path is proposed in this paper. This method can reduce computation amount and fasten search process to a great extent.

\author{
Xin Zhou \\ Xi'an Research Institute of High-tech \\ Shaanxi, China \\ Shanni, China
}

\section{II. $\triangle$-NEIGHBORHOOD OF EXPECTED SOLUTION}

The search area is usually determined in this way: the centre is INS track, and we extend the center along the longitude and latitude to a rectangle. Size of the rectangle is determined by the largest estimate of INS error to assure the real track is inside the map. Information including geographic latitude, geographic longitude, and geomagnetic field of the search area is stored in computer beforehand, which is so-called reference map. Because INS has no heading error in a brief time, we assume INS track parallels vehicle track in matching time, and the latter (what is called real-time track) is the expected solution of matching. Assuming that the effective matching area in reference map is square grid $M$ by $M$. Every grid in the reference map may be the starting point of the track. Once the starting point is fixed, other points in the track are known according to information from INS. All the paths with geomagnetic field intensity preparing to match are named sub-maps. If reference map has $\mathrm{M} \times \mathrm{M}$ grids, the number of sub-maps is $\mathrm{S}=\mathrm{M} \times \mathrm{M}$. Assumed $N$ is the length of sampling sequence of real-time track, matching is the process of finding out the only one path which is the closet to real-time geomagnetic field intensity sequence $\left\{T_{t}(k), k=1,2, \cdots, N\right\}$ in the sub-maps $\left\{T_{j}(k), k=1,2, \cdots N\right.$, $j=1,2, \cdots, S\}$. "Close" can be measured by mean absolute difference (MAD) described in expression (1):

$$
\mathrm{D}(j)=\frac{1}{\mathrm{~N}} \sum_{k=1}^{\mathrm{N}}\left|\mathrm{T}_{j}(k)-T_{t}(k)\right|
$$

The sub-map which minimizes measurement (1) is the result we search. As a common geomagnetic matching method, MAGCOM find out the result by comparing the measurement all over the solutions. Consequently, amount of computation will increase rapidly with INS error increasing. To use geomagnetic matching in this situation, we must reduce the number of search solutions while search area is unchanged. 
In matching time $\left[t_{1}, t_{N}\right]$, the distance between two continuous curves $T_{i}(t)$ and $T_{j}(t)$ is defined as:

$$
\begin{aligned}
& d_{0}=\max \left|T_{i}(t)-T_{j}(t)\right|, \\
& d_{1}=\max \left|T_{i}^{\prime}(t)-T_{j}^{\prime}(t)\right|
\end{aligned}
$$

$d_{0}$ and $d_{1}$ satisfy distance axiom, and they are named the zero order distance and the first order distance separately. Let the geomagnetic field continuous curves in the track of vehicle is $\hat{T}(t)$, and the sampling sequence of the same track in reference map is $\hat{T}(k)$. In order to decrease search scope, we can choose to search in the neighborhood of expected solution only. $\hat{T}(k)$ is just the expected solution. Choosing the belt from $\hat{T}_{j}(t)+\delta$ to $\hat{T}_{j}(t)-\delta$ as the solutions to search (as is shown in Fig .1. $\mathrm{Y}$-axis is geomagnetic field intensity, and $\mathrm{X}$-axis is time.) If one geomagnetic curve in track $T_{j}(t)$ is inside the belt, it means that the distance between $T_{j}(t)$ and $\hat{T}_{j}(t)$ is less than (or equals) $\delta . \delta$ can be defined as the zero order or the first order distance. The belt is named $\delta$-neighborhood of measurement $\hat{T}_{j}(t)$, which is less than rectangle search area obviously.

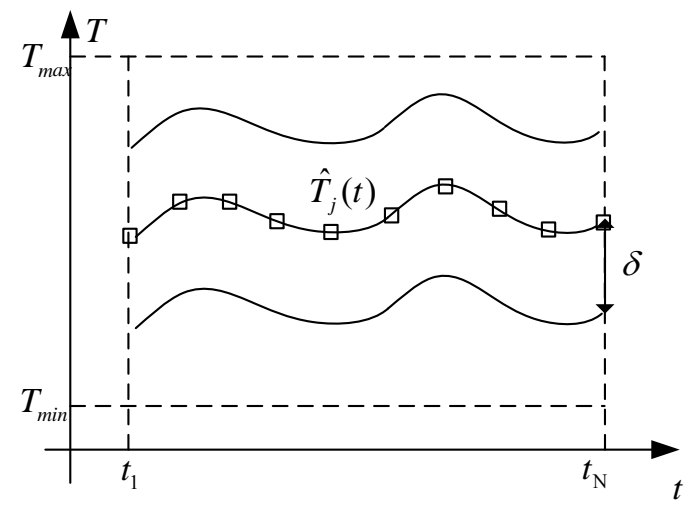

Figure 1. distance between geomagnetic field continuous curves

\section{SEARCHING IN $\Delta$-NEIGHBORHOOD}

Notice that the purpose $\hat{T}_{j}(t)$ can not be obtained beforehand. In the following, two solutions to confirm $\delta$ neighborhood are presented.

\section{A. Ideal condition}

In this case, no any error exists between reference map and real-time measurement sequence, that is $\hat{T}_{j}(k)=T_{t}(k)$.Therefore, the search area is $\delta$ neighborhood of $T_{t}(k)$. Keeping sampling time unchanged, $\delta$ is the threshold value to express the size of neighborhood.
The search process is described as follows: After start point $T_{t}(1)$ is measured, we search all the tracks in which sub-map is inside the area $\left|T_{t}(1)-T_{j}(1)\right| \leq \delta_{0}$.As the zero order distance, $\delta_{0}$ is the threshold of measurement intensity error permitted. That's to say, $\delta_{0}$ is the width of error belt whose center is $T_{t}(1)$. The tracks outside the area are excluded. With the vehicle moving, we can get the next measurement $T_{t}(2)$, and compute the average change of rate $K_{t}(1)$ of two geomagnetic field intensity, and search the tracks satisfying $\left|T_{t}(2)-T_{j}(2)\right| \leq \delta_{0}$ and $\left|K_{t}(1)-K_{j}(1)\right| \leq \delta_{1}$ in present solutions, where $\delta_{1}$ is the threshold of measurement grads error permitted. This process which excludes the impossible tracks continues until one of the following conditions satisfies: only one track left; finding out the track minimum measurement in the left sub-map.

Not considering measurement error, $\hat{T}_{j}(k)$ is superposition of $T_{t}(k)$. In this case, let $\delta_{0}=0$, and $\delta_{1}=0$. Since $\hat{T}_{j}(k)$ must be in the sub-map, we can find the real track of the vehicle.

\section{B. Effect of error}

Considering errors between real-time geomagnetic field intensity sequence and reference map, $\hat{T}_{j}(k)$ does not accord with $T_{t}(k)$ any more, and the corresponding distance between them is $\delta_{0}>0 \delta, \delta_{1}>0$. We must choose $\delta=\max \left(\delta_{0}, \delta_{1}\right)$ large enough so that expected solution is in the $\delta$-neighborhood of $T_{t}(k)$. However, large $\delta$ may increase matching time. On the other hand, small $\delta$ may mistake the tracks because the expected solution may be outside the search area. Therefore, in order to reduce the computation and increase the precision, the most important issue is choosing threshold $\delta$ appropriately.

The error between the reference map and real-time geomagnetic field intensity sequence can be described in three forms: Gauss white noise distributed with mean 0 and variance $\sigma^{2}$, noise uniformly distributed in $[-a / 2, a / 2]$, and constant noise with value $\mu$. Assume that these three kinds of noise do not correlate and satisfy additive description. Take $3 \sigma$ as bound of Gauss white noise and assume sampling period as per unit, then distance $\delta$ can be chosen as

$$
\delta \leq 3 \sigma+a
$$

Since the quantity in the measurement performs the arithmetic operation of subtraction, constant noise will not bring difference.

Choosing (3) as criterion for search area has a disadvantage. In (3), we look $3 \sigma$ as distributed range of white Gauss noise with mean 0 and variance $\sigma^{2}$. 
According to statistical probability, the real-time geomagnetic field intensity is very likely in this range. However, there are still individuals outside the range which are called wild points.

If there are wild points in real-time sequence, the expected solution, which correlates with vehicle track, may be excluded from possible solutions. It will lead searching to failure. To avoid this situation, we refer to[5], and have operation as follows:

After getting several measurement data, we compute the average value $\bar{\delta}$.If $\bar{\delta} \geq 3 \sigma+a$, the solution is excluded. The more measurement data we compute, the better possible solutions, because the influence of the wild points will be less.

\section{SimUlation OF THE $\Delta$-NEIGHBORHOOD MAGNETIC CONTOUR MATCHING ALGORITHM}

Before In the following simulation, the reference maps have $60 \times 60$ grids. The noise is composed of uniform and Gauss ones. The former is uniformly distributed in $[-10,10]$, and the later is with mean 0 and variance $\sigma^{2}=20$, then $\delta=46.8$.After 1000 times simulation in various matching length, we can get matching probability and errors of $\delta$-neighborhood MAGCOM, and compare these quality indexes with standard MAGCOM, as shown in Fig .2.

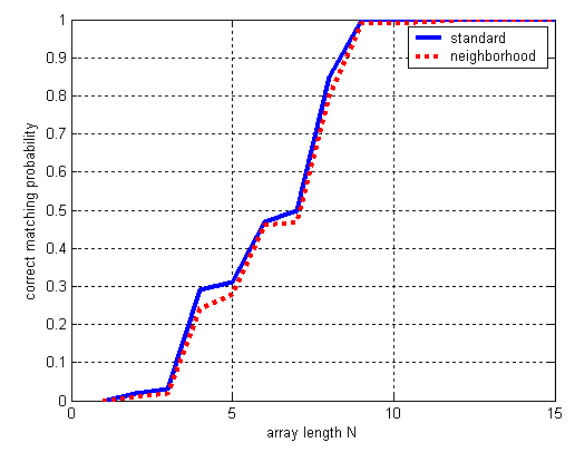

(a)Comparison of matching probability in various matching length

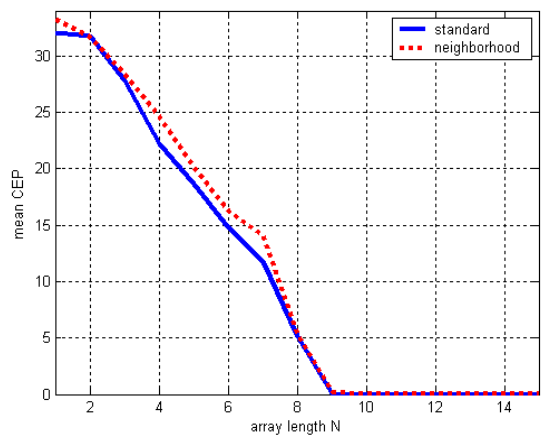

(b) Comparison of location errors in various matching length Figure. 2 Comparison of matching probability and precision in searching all over the solutions and in $\delta$-neighborhood in various matching length

From Fig .2, we can see matching probability and position error of $\delta$-neighborhood MAGCOM are a bit worse than standard algorithm. It is mostly because we get local optimum solution when we search in $\delta$-neighborhood.
Meanwhile, if we replace the real geomagnetic field by measurement one, position may be failure because of the real vehicle track outside the searching area. The difference between searching area in two algorithms can be decreased by enlarging $\delta$. In the simulation (Fig .2), the maximum difference of mean matching probability is $5 \%$, and the maximum difference of position error is 2.258 grids. Moreover, when matching array length grows to a certain number, matching probability in two algorithms can be $100 \%$. It means choosing $\delta \leq 3 \sigma+a$ is suitable.

On the other hand, the main purpose of searching in neighborhood is to shrink the search area and reduce matching time. In the following, we will compare the timecosting performance of two algorithms. Simulation parameters are the same as the former. In various matching array length, time-costing for two algorithms is as shown in Fig .3. In standard algorithm, since the search area is all over the field, the number of match array is certain. Matching time will be increases almost linearly with the length of sequence $\mathrm{N}$. However, the computation of searching in neighborhood rests with the number of possible solutions in $\delta$. Since noise is random, the number of possible solutions in $\delta$ is not fixed. Therefore, matching time is different even in the same length of sequence. In order to represent time-costing, we use three curves: mean, maximum and minimum. From Fig .3 , we can see the maximum time-costing may be very long, because wild points would bring the possible solutions in $\delta$ to be zero, which need longer $\delta$ neighborhood and search again. With the length of matching sequence $\mathrm{N}$ increasing, because the possible solutions will decrease quickly, the presented algorithm has advantage in computation efficiency.

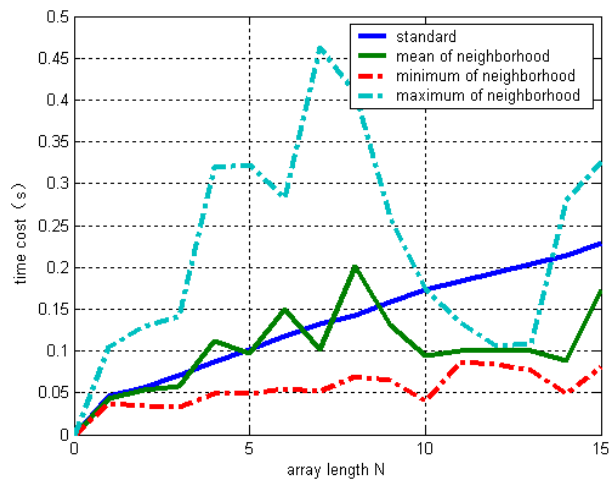

Figure.3 Time-cost of searching all over the solutions and in $\delta$ neighborhood in various matching length

\section{CONCLUSION}

In this paper, we present an improved geomagnetic contour matching algorithm, which can achieve lower time-cost by decrease search area to $\delta$-neighborhood. The size of $\delta$ is discussed in ideal and error condition. At last, we evaluate the effect of improved algorithm by simulation. The results show that this algorithm has advantage in computation efficiency, and doesn't make matching probability and position error worse obviously. At the same time, the algorithm is more appropriate to the situation that matching sequence $\mathrm{N}$ is long enough and wild points in measurement sequence have been pretreated. 


\section{REFERENCES}

[1] Wang Peng, Zhang Yingchun. Method of high precision autonomous navigation based on geomagnetic. Systems Engineering and Electronics, 2012,34(8):1160-1167

[2] Xu Yu, Ren Qinyuan, Sun Wenda, Li Ping. A geomagnetic navigation algorithm for miniature unmanned helicopter. Acta Armamentarii, 2011,32(3):337-342.

[3] Huang Bin, Sun Yongrong, Wang Lina, Liu Jianye, Gao Xiaoying. Iterative evaluation matching algorithm for geomagnetic navigation. Journal of NanJing University of aeronautics and astronautics,2012,44(4):565-569

[4] Zhou Jun, Ge Zhilei, Shi Guiguo, Liu Yuxia. Key technique and development for geomagnetic navigation. Journal of Astronautics,2008,29(5):1467-1471.

[5] Yue Yazhou, Lu Zhidong, Dang Jin, Gao Guangen. Terrain matching algorithm based on path screening. Journal of Chinese Inertial Technology, 2009,17(5):532-537.
[6] HUANG Feng, WANG Yue-feng, WANG Jin-yu, NIU Yan-xiong Study on application of high-repetition-rate solid state lasers in photoelectric countermeasure[J]. Infrared and Laser Engineering, 2003, 32(5):465-467. (in Chinese)

[7] XING Hui, LEI Ping, MA Na. Experiment and analysis on time sequence anti-jamming technology of semi-active laser seeker[J] Infrared and Laser Engineering, 2012, 41(2): 461 465. (in Chinese).

[8] Kneizys F X, et al . Atmospheric Transmittance/Radiance Computer Code LOWTRAN 6 [R]. Report AFGL-TR-83-0187, Air Force Geophysics Laboratory, 1983.

[9] Catrter J M. New HWIL motion system developments[J]. Proc.SPIE .2001, 4366 : 194-203.

[10] Eguchi H ,Obana K , Kamiya M. Hardware-in-the-loop missile simulation facility[J]. Proc.SPIE. 1998,3368:2-9. 